\title{
Not Remembering History, Dooms Us to Repeat It: Using the Lessons of the Global HIV Response to Address COVID-19
}

\author{
Mary Jane Rotheram-Borus ${ }^{1}$ (D) $\cdot$ Mark Tomlinson $^{2,3}$
}

Accepted: 12 October 2020 / Published online: 29 October 2020

(c) Springer Science+Business Media, LLC, part of Springer Nature 2020

\begin{abstract}
High income countries (HIC) have set the initial global policy responses to COVID-19. Yet, low and middle income countries (LIMIC) face very different challenges than HIC. In LMIC, there is a far greater emphasis on community solutions; families live in far more dense communities, making shelter-in-place mandates questionable; and strengthening existing health systems is more important than novel services. LMIC have far fewer economic resources. Most distressing, the successful economic commitments that HIC made to help stop HIV in LMIC have not yet been imitated, or even initiated-this support is needed now to fight COVID-19.
\end{abstract}

Keywords COVID-19 $\cdot$ High income countries $\cdot$ HIV $\cdot$ Prevention $\cdot$ Public health

\section{Guest Editorial}

Global health in 2020 has been dominated by COVID-19 and high-income countries (HICs) are setting most of the priorities [1]. Currently, two-thirds of the headquarters of global organizations involved in health and health policy are located in only three HICs (USA, UK and Switzerland). Drawing on decades of lessons from the HIV epidemic, we argue in this commentary that LMICs need to design and implement their own COVID policies, to build on their strengths and also to have the courage to resist policy and financial directives from HICs.

\section{Individual vs Community Focus}

Driven by HIC, HIV's initial solutions were almost always protecting individuals. In 1985, the USA set guidelines for individual, confidential, voluntary pre- and post-test HIV

Mary Jane Rotheram-Borus

CCHPublications@mednet.ucla.edu

1 University of California, Los Angeles, USA

2 Department of Global Health, Institute for Life Course Health Research, Stellenbosch University, Cape Town, South Africa

3 School of Nursing and Midwifery, Queens University, Belfast, UK counseling (VCT), aiming to avoid potential HIV stigma to already stigmatized groups (e.g., injecting drug users). HICs soon adopted these guidelines and funding in LMIC was contingent on adopting a VCT approach. When HIV treatments became available, in 1998 the USA and other HIC eliminated pre- and post-test counseling for high risk groups (e.g. pregnant women). Yet, the recommendation of individual VCT was maintained by the World Health Organization through 2019 [2]. Getting counselled communicates a problem in one's sexual behavior [3] and HIV remains a problem of 'infected' people 'responsible' for 'infecting' fellow community members. In LMIC, acquiring HIV is an issue of geography_living in a high prevalence community confers risk. Individual testing breaks down the sense of family and community responsibility, as there is no established process for sharing test results; family secrets emerge [4].

Many LMICs have a much greater "group orientation" and focus on community responsibility [5]. This is critical when selecting prevention strategies, as well as in deciding how to allocate resources. When HIC adopted VCT and an HIV care system, the policies preserved the rights and served the needs of the individual, and less so, the community. These policies created both economic drains and lost opportunities for LMICs. For VCT, a new workforce of counselors was created, draining both talent and resources which could have been deployed elsewhere. It is critical that we not perpetuate this tendency in COVID-19 testing. 


\section{HIV Exceptionalism}

It was easier for HIC to highly train a subset of professionals to treat patients with complex HIV infections, rather than spread responsibility for persons with HIV throughout the health system. HIV stand-alone clinics and hospital wards were created to deliver HIV-related services. The practice extended to LMICs, where a vertically-integrated HIV system of care was established. HIV also drained attention from other health priorities-malaria, TB-a controversial decision since the burden of HIV disease was often lower than other health challenges in LMIC [6].

Rather than creating new COVID-19 services, this pandemic may provide the opportunity to strengthen LMICs' health systems. COVID-19 is already expected to lead to an additional 673,000 HIV-related deaths (medication not being picked up), 382,000 malaria deaths, and 2.3 million child deaths this year [7]. In contrast, COVID-19 care could potentially create opportunities to strengthen existing healthcare systems, especially if focusing on community-based care [8].

\section{Safety Nets}

HICs have a variety of safety nets that can be employed during the outbreak of an epidemic or a pandemic. Early in the HIV epidemic, USA Ryan White funding provided rent, at-home health care, and income replacement for families coping with HIV. Similarly, the USA was able to pass a $\$ 2$ trillion budget for immediate COVID relief and made it illegal to evict anyone for not paying rent; mortgage loan repayments were suspended; and food distribution sites were established with no means testing.

Without the safety nets of HIC, lockdown is not a solid public health strategy for most LMICs-large numbers of people live-in high-density households, and more than $50 \%$ of the population earn a living through informal work. The economic consequences are life-threatening for LMIC. As one example, in May and June of this year (first 2 months of lockdown), food insecurity went from 8.3 to $51.8 \%$ in rural Bangledesh compared to 2 years ago [9]. In addition, more than 370 million of the 1.8 billion children out-of-school in LMIC have lost their school meals, for many, a primary source of nutrition [10].

Perhaps, it may be better for communities to shelterin-place with a larger circle of trusted members than is necessary in HIC. This would also allow batch testing for COVID, rather than individual testing. This would also involve engaging with existing and new community organizations to protect neighborhood health, help ground prevention messages in local community contexts, and in so doing also reduce stigma.

\section{The Guarantee of Treatments and Vaccines from HIC}

The financing of treatments has been one area in which HIV needs to become the global standard when dealing with COVID-19. Pharmaceutical companies in HICs originally balked at the idea of giving discounts to LMIC for costly HIV medications and, in fact, sued South Africa to stop the use of generics [11]. Today, drug companies charge Africa \$75 annually for drugs for persons living with HIV, while charging the USA $\$ 39,000$ [12]. The cost of pre-exposure prophylaxis range from $\$ 8 /$ month in Australia to $\$ 1,600 /$ month in the U.S [13]. With COVID ventilators costing $\$ 25,000$ to $\$ 50,000$ and the USA cornering the world's supply of Remdesivir at a cost of $\$ 3,200 /$ course of treatment-LMIC are left undefended [14].

HIV has provided the model for discount medications and vaccines identified in HIC when delivered to LMIC. The global networking necessary to achieve this bargain has not appeared to begin, however.

\section{Conclusion}

As LMICs struggle to cope with COVID-19, there dependence on support from HIC will continue. With the economies of HIC being concurrently stressed, it will likely be more difficult to both sustain and/or expand their support of LMIC. Yet, as the HIV epidemic has evolved, more and better local solutions emerged. COVID-19 creates an opportunity for LMICs to differentiate themselves with more locally efficient, public health policy alternatives and to 'build back better'. COVID-19 solutions simply cannot be a 'one size fits all approach'. We encourage innovative local solutions that build on LMICs' existing strengths. There are many possibilities now; let's hope we choose good pathways.

Author Contributions All authors have contributed equally to the commentary.

Funding No funding to report.

\section{Compliance with Ethical Standards}

Conflict of interest The authors have no conflicts of interest to report. 


\section{References}

1. Walker PGT, Whittaker C, Watson OJ, Baguelin M, Winskill P, Hamlet A, et al. The impact of COVID-19 and strategies for mitigation and suppression in low- and middle-income countries. Science. 2020. https://doi.org/10.1126/science.abc0035.

2. WHO. HIV/AIDS: World Health Organization; 2020. Available from: https://www.who.int/news-room/fact-sheets/detail/hiv-aids.

3. Steinberg J. Three-letter plague: a young man's journey through a great epidemic. Johannesburg: Jonathan Ball Publishers; 2008.

4. van Rooyen H, Essack Z, Rochat T, Wight D, Knight L, Bland R, et al. Taking HIV testing to families: designing a family-based intervention to facilitate HIV testing, disclosure, and intergenerational communication. Front Public Health. 2016;4:154.

5. Miyamoto Y, Yoo J, Levine CS, Park J, Boylan JM, Sims T, et al. Culture and social hierarchy: self- and other-oriented correlates of socioeconomic status across cultures. J Pers Soc Psychol. 2018;115(3):427-45.

6. Amico P, Aran C, Avila C. HIV spending as a share of total health expenditure: an analysis of regional variation in a multi-country study. PLoS ONE. 2010;5(9):e12997.

7. Roberton T, Carter ED, Chou VB, Stegmuller AR, Jackson BD, Tam Y, et al. Early estimates of the indirect effects of the COVID19 pandemic on maternal and child mortality in low-income and middle-income countries: a modelling study. Lancet Glob Health. 2020;8(7):901-8.
8. Cash R, Patel V. Has COVID-19 subverted global health? Lancet (London, England). 2020;395(10238):1687-8.

9. Hamadani JD, Hasan MI, Baldi AJ, Hossain SJ, Shiraji S, Bhuiyan MSA, et al. Immediate impact of stay-at-home orders to control COVID-19 transmission on socioeconomic conditions, food insecurity, mental health, and intimate partner violence in Bangladeshi women and their families: an interrupted time series. Lancet Glob Health. 2020. https://doi.org/10.1016/S2214-109X(20)30366-1.

10. Saavedra J. blogs.worldbank.org: World Bank. 2020. [cited 2020]. Available from: https://blogs.worldbank.org/education/educationa 1-challenges-and-opportunities-covid-19-pandemic.

11. Sidley P. Drug companies sue South African government over generics. BMJ. 2001;322(7284):447.

12. Rosenberg T. H.I.V. drugs cost $\$ 75$ in Africa, $\$ 39,000$ in the U.S does it matter? The New York Times. 2018, September 18

13. Salzman S. What does PrEP cost outside the United States?: The Body; [updated 2019, July 3. Available from: https://www.thebo dy.com/.

14. Rowland C. More lifesaving ventilators are available. Hospitals can't afford them. Washington Post. 2020, March 18.

Publisher's Note Springer Nature remains neutral with regard to jurisdictional claims in published maps and institutional affiliations. 